\title{
Comparing the Utility of the Theory of Planned Behavior Between Boys and Girls for Predicting Snack Food Consumption: Implications for Practice
}

\author{
Paul Branscum, PhD, $\mathrm{RD}^{1}$ \\ Manoj Sharma, PhD, MBBS $^{2}$
}

The purpose of this study was to use the theory of planned behavior to explain two types of snack food consumption among boys and girls (girls $\mathrm{n}=98$; boys $\mathrm{n}=69$ ), which may have implications for future theorybased health promotion interventions. Between genders, there was a significant difference for calorie-dense/ nutrient-poor snacks ( $\mathrm{p}=.002)$, but no difference for fruit and vegetable snacks. Using stepwise multiple regression, attitudes, perceived behavioral control, and subjective norms accounted for a large amount of the variance of intentions (girls $=43.3 \%$; boys $=55.9 \%$ ); however, for girls, subjective norms accounted for the most variance, whereas for boys, attitudes accounted for the most variance. Calories from calorie-dense/ nutrient-poor snacks and fruit and vegetable snacks were also predicted by intentions. For boys, intentions predicted $6.4 \%$ of the variance for fruit and vegetable snacks ( $\mathrm{p}=.03$ ) but was not significant for caloriedense/nutrient-poor snacks, whereas for girls, intentions predicted $6.0 \%$ of the variance for fruit and vegetable snacks $(\mathrm{p}=.007)$, and $7.2 \%$ of the variance for calorie-dense/nutrient-poor snacks ( $\mathrm{p}=.004)$. Results suggest that the theory of planned behavior is a useful framework for predicting snack foods among children; however, there are important differences between genders that should be considered in future health promotion interventions.

Keywords: child/adolescent health; health education; behavior change theory; theory; nutrition

Health Promotion Practice

January 2014 Vol. 15, No. 1 134-140

DOI: $10.1177 / 1524839913481974$

(C) 2013 Society for Public Health Education

\section{-INTRODUCTION}

Effective and innovative behavior change interventions are greatly needed to improve society's health and well-being. Such interventions can occur on a number of levels, including the intrapersonal (e.g., enhancing an individual's attitudes toward being physically active), interpersonal (e.g., providing social support to assist an individual with smoking cessation), and societal level (e.g., passing laws that limit blood alcohol concentration when driving). No matter what level implementation occurs however, behavior change theories should be used and operationalized in program planning, implementation, and evaluation. Theories are essential for understanding behavior change as they give guidance for describing and understanding the behavior change process. To improve our understanding of the effectiveness, or ineffectiveness, of theoretical frameworks as they relate to health behaviors, it is necessary to empirically test them under varying settings and circumstances.

The theory of planned behavior (TPB) has prominence as one of the most popular theories used in health promotion and health education. In 2010, it was reported that the research program of Icek Ajzen, a creator of the TPB, had the highest scientific impact among 62 of the top social psychologists in the United States and Canada (Nosek et al., 2010). TPB has been used to predict a number of health behaviors, including

${ }^{1}$ The University of Oklahoma, Norman, OK, USA

${ }^{2}$ The University of Cincinnati, Cincinnati, OH, USA

Authors' Note: We would like to thank the physical education staff in the Princeton City School District, Cincinnati, OH. Please address correspondence to Paul Branscum, PhD, RD, Department of Health and Exercise Science, The University of Oklahoma, 1401 Asp Avenue, Norman, OK 73019, USA; e-mail: pbranscum@ou.edu. 
those related to nutrition and physical activity (Ickes \& Sharma, 2011; Kassem, Lee, Modeste, \& Johnston, 2003; Martin, Oliver, \& McCaughtry, 2007), as well as substance abuse (Conner \& Mcmillan, 1999) and sexual behaviors (Beadnell et al., 2007). TPB is predicated on the fact that behavior is primarily predicted by behavioral intentions. In turn, intentions are influenced by three behavioral constructs, including attitudes toward the behavior, or the overall positive or negative evaluation of a behavior, subjective norms, or the belief that influential people in one's life approves or disapproves of engaging in a behavior, and perceived behavioral control, or whether an individual feels the behavior is within their own control, and they feel confident they can act on their own behalf (Glanz, Rimer, \& Viswanath, 2008).

Health promotion and health education interventions that address the issue of childhood obesity prevention are greatly needed. Currently, one out of every three children is considered overweight, and one out of every six is obese (Ogden, Carroll, Kit, \& Flegal, 2012). Gender differences have also emerged. Although there were no differences in overall obesity rates among boys and girls from the last five National Health and Nutrition Examination Survey reports (Ogden, Carroll, Curtin, Lamb, \& Flegal, 2010; Ogden, Carroll, \& Flegal, 2008), authors from the most recent report found that boys had a significantly higher obesity rate than girls (Ogden et al., 2012). Differences in dietary intake have also been shown between genders. Using a nationally representative sample of children and adolescents, authors found that on average boys consumed a higher amount of calories per day than girls, and the average portion size and energy content of meals and snacks were higher in boys, compared with girls (Huang, Howarth, Lin, Roberts, \& McCrory, 2004). Another study showed boys consume significantly less fruits and vegetables than girls (Bere, Brug, \& Klepp, 2007).

A number of dietary behaviors have been found as determinants of obesity, such as the consumption of sugar-sweetened beverages, fruits and vegetables, and energy-dense foods (Barlow \& The Expert Committee, 2007). The foods we consume can typically be categorized as being part of meals (breakfast, lunch, and dinner) or as snack foods (foods consumed outside of the three meals). Since meals are typically more structured eating occasions, whereas snacks are often less structured and planned, replacing unhealthy snacks, such as those with high energy density, with healthier snacks, such as fruit and vegetable snacks, is a viable target for public health interventions. The purpose of this study was twofold. The first purpose was to report any differences in the intake of calorie-dense/nutrient-poor snack foods, and fruit and vegetable snack foods among boys and girls. The second purpose was to explore the efficacy or utility of the TBP for predicting both types of snack foods among boys and girls.

\section{METHOD}

\section{Study Sample}

Children enrolled in the fourth and fifth grade from a Midwestern public school district were eligible for this study. This district was racially diverse, and included a majority of White (58\%), and African American students (35\%), and almost equal number of males and females. Enrollment began with the corresponding author first explaining to children the purpose of the study and the importance of having one parent sign and return a parent permission form. Subsequently, children were asked to sign an informed assent form. During this procedure, children were reminded that participation was strictly voluntary, and the answers they provided would be anonymous. Surveys were administered in a gym classroom setting, with approximately 15 to 20 children per class. The The University of Cincinnati Institutional Review Board approved this study.

\section{Instrument}

The instrument used in this study contained three sections, with each section measuring (a) snack food consumption, (b) demographic information, and (b) TBP constructs. To evaluate snack food consumption, children were asked to report and record all foods they consumed in the previous 24 hours between breakfast, lunch, and dinner. Children were also instructed to be as specific as possible for each food they listed and were encouraged to include important features of the food such as approximate portion sizes and brand names. This was further simplified by breaking each time period down (e.g., the time between lunch and dinner), and probing children with follow-up questions to heighten their memory (e.g., probing for hidden foods). Snack foods of interest included calorie-dense/ nutrient-poor snacks, such as cookies, candy, and fried potato chips, and fruit and vegetable snacks, including all types (fresh, frozen, and canned), but not including juices. Snack consumption was quantified by entered all foods into the U.S. Department of Agriculture National Nutrient Database for Standard Reference (Release 22), recording the number of calories each food contained and summating total calories for each snack type. Calories were used as a dependent variable in this study instead of the number of portions or 
serving sizes of snacks, to help increase the variability of both variables. Also, calories were thought to be a sensitive indicator of consumption, as it accounts for both the type of snack food consumed and the snack's portion size. Demographic questions included race or ethnicity, gender, grade, and age.

To measure the TPB constructs, the principal investigator implemented the Theory of Planned Behavior \& Snacks Questionnaire, which has been described elsewhere (Branscum \& Sharma, 2011-2012). In short, this portion of the survey contained 13 questions, all of which were measured on a 7-point sematic differential scale. Three questions were used to measure behavioral intentions, subjective norms, and perceived behavioral control (making a possible score range of 3 to 21 for each construct), and four questions were used to measure attitudes (making a possible score range of 4 to 28). This survey was also shown to have adequate validity, as measured by a panel of experts (face and content validity) and factor analysis (construct validity) and reliability, as measured by Cronbach's alpha (internal consistency reliability) and administering the survey twice to a group of students (test-retest reliability).

\section{Data Analysis}

All data were analyzed by SPSS (Version 19.0). Frequencies, means, and standard deviations were first used to summarize all responses. Independent $t$ tests were used to examine differences in key variables between boys and girls. To evaluate the predictive nature of the constructs of the theory, stepwise multiple regression was used in two rounds. First, attitudes, subjective norms, and perceived behavioral control scales predicted intentions. Second, intentions predicted calories from both types of snack foods (caloriedense/nutrient-poor snacks, and fruit and vegetable snacks). An a priori criterion to enter each model was set at an alpha of .05 and the criterion to be removed from the model was an alpha of .10. To predict a medium to large effect size (McEachan, Conner, Taylor, \& Lawton, 2011) with three predictor variables, an alpha of .05 and power of $80 \%$, an a priori sample size of 59 was determined for both groups in this study ( $\mathrm{G}^{*}$ Power, Version 3.1.3).

\section{RESULTS}

Overall, 98 girls ( $M$ age $=10.5$ years) and 69 boys $(M$ age $=10.3$ years) were enrolled in this study. Prior to data analysis, data were examined for missing values and normality. For inclusion in this study children were required to complete at least $80 \%$ of all questions, and this was confirmed among all surveys. In rare cases that children missed select questions, the mean replacement method was employed. Next, all variables were confirmed as being normally distributed by using measures of skewness and kurtosis. On data analysis, outliers were examined by use of Cook's distance and multicollinearity was examined by use of variance inflation factor. For both regression models, no issues were found for either outliers or multicollinearity.

Independent group $t$ tests were used to examine gender differences for TPB constructs and both types of snacking behaviors. Results showed that calories from calorie-dense/nutrient-poor snacks were significantly higher ( $p=.002)$ among girls $(M=348.6$ calories) than boys ( $M=240.9$ calories), but not significantly different for calories from fruit and vegetable snacks (boys $M=$ 49.9 calories; girls $M=46.1$ calories). Intentions, attitudes, and subjective norms also did not significantly differ between the genders; however, boys $(M=17.1)$ reported significantly $(p=.04)$ higher perceived behavioral control than girls $(M=16.0)$.

For the first model, intentions were predicted by attitudes, perceived behavioral control, and subjective norms. For both boys and girls, all variables were significant and entered the model; however, the amount of variance explained by each construct for intentions varied. Collectively for boys, all three variables explained $55.9 \%$ of the variance of intentions. Attitudes $(p<.001)$ was entered into the model first, which explained an initial $43.3 \%$ of the variance, followed by perceived behavioral control ( $p=.003$ ), which added $10.0 \%$ to the total variance, and finally subjective norms $(p=.03)$ added $2.6 \%$ of the variance. Collectively for girls, all three variables explained $39.3 \%$ of the variance of intentions. Subjective norms $(p<.003)$ was entered into the model first, explaining an initial $26.6 \%$ of the variance, followed by perceived behavioral control ( $p=.006)$ adding $8.3 \%$ to the total variance, and finally attitudes ( $p=$ .006 ) added $4.4 \%$ of the variance (Table 1 ).

For the second model, calories from calorie-dense/ nutrient-poor snacks and fruit and vegetable snacks were predicted by intentions. For boys, intentions predicted $6.4 \%$ of the variance for fruit and vegetable snacks $(p=.03)$ but was not significant for caloriedense/nutrient-poor snacks. For girls, intentions predicted $6.0 \%$ of the variance for fruit and vegetable snacks $(p=.007)$, and $7.2 \%$ of the variance for caloriedense/nutrient-poor snacks ( $p=.004$; Table 1).

\section{DISCUSSION}

The purpose of this study was to explore possible gender differences for the predictive nature of the TPB, 
TABLE 1

Parameter Estimates and Model Prediction for Boys and Girls Snacking Behaviors

\begin{tabular}{|c|c|c|c|c|}
\hline & $\mathrm{R}^{2}$ & $\begin{array}{l}\text { Standardized } \\
\text { Coefficients } \beta\end{array}$ & $\mathrm{t}$ & $\mathrm{p}$ \\
\hline Model 1 (boys): Predicting INT & .559 (total) & & & \\
\hline Attitudes & .433 & .568 & 6.75 & .000 \\
\hline PCB & .100 & .277 & 3.11 & .003 \\
\hline Norms & .026 & .188 & 2.21 & .030 \\
\hline Model 1 (girls): Predicting INT & .393 (total) & & & \\
\hline Norms & .266 & .291 & 3.10 & .003 \\
\hline PCB & .083 & .272 & 2.84 & .006 \\
\hline Attitudes & .044 & .250 & 2.82 & .006 \\
\hline Model 2 (boys): Predicting FV Int & .06 & .272 & 2.28 & .030 \\
\hline Model 2 (girls): Predicting FV Int & .06 & .271 & 2.75 & .007 \\
\hline Model 2 (girls): Predicting CDNP Int & .072 & -.286 & -2.92 & .004 \\
\hline
\end{tabular}

NOTE: INT = intentions; PCB = perceived behavioral control; Norms = subjective norms; FV = fruit and vegetable snacks; CDNP = calorie-dense/nutrient-poor snacks.

as it relates to consuming energy-dense/nutrient-poor snacks and fruit and vegetable snacks. For both boys $(55.9 \%)$ and girls (39.3\%), intentions was significantly predicted by attitudes, subjective norms, and perceived behavioral control, which confirms the model's usefulness. These results were similar to a meta-analysis recently published on the TPB, which found attitudes, subjective norms, and perceived behavioral control on average account for $44.3 \%$ of the variance of intentions across multiple health behaviors (McEachan et al., 2011). The meta-analysis also found that correlations between the three variables and intentions ranged from 0.40 to 0.57 , which is similar to our results with two exceptions: For boys, the correlation between subjective norms and intention was 0.33 and between attitudes and intentions was 0.66 (McEachan et al., 2011; Figure 1). Authors from the same meta-analysis also noted that attitudes were the strongest predictor of intentions, followed by perceived behavioral control, and finally subjective norms. This was also observed in this study among the boys but not among girls. In fact, the opposite was true for the girls; subjective norms predicted a majority of the intentions, and attitudes added only a small, yet significant, amount of variance. This was similar to a study using the theory of reasoned action to predict sweet snack food consumption among men and women in the United Kingdom. Attitudes were found to be a significant predictor for both men and women; however, subjective norms were only significant for women (Grogan, Bell, \& Conner, 1997).
This study suggests that different behavioral antecedents shape boys' and girls' intentions toward healthy and unhealthy snacking behaviors; however, a large amount of the variance of intentions remains unexplained in both cases. Recently, Ajzen (2011) published an editorial elaborating on this issue, that additional predictors of intentions may be needed, and identified the two most commonly proposed variables as self-identity and anticipated affect. Self-identity has been referred to as prominent and stable aspects of one's self-perception, and often is evaluated by completing statements such as "I am . ..." For example, one might see oneself as "a mother," "a student," "an honest person," or in the context of health, "a healthy eater" or "a physically active person.” A meta-analysis showed that inclusion of self-identity on average explains an additional $6 \%$ of the variance of intentions, controlling for the three traditional behavioral antecedents of the TPB and an additional $9 \%$ of the variance of intentions when additionally controlling for past behaviors (Rise, Sheeran, \& Hukkelberg, 2010). This appears to be a worthwhile construct for exploring in future research.

The other predictor Ajzen (2011) noted was anticipated affect, which refers to emotional states one anticipates feeling after engaging in a behavior. In the context of this study, although children may have positive attitudes toward eating fruit and vegetables as snacks, they could anticipate that the snacks will not taste as good as high-calorie snacks, having a negative anticipated affect, or they could anticipate that eating 


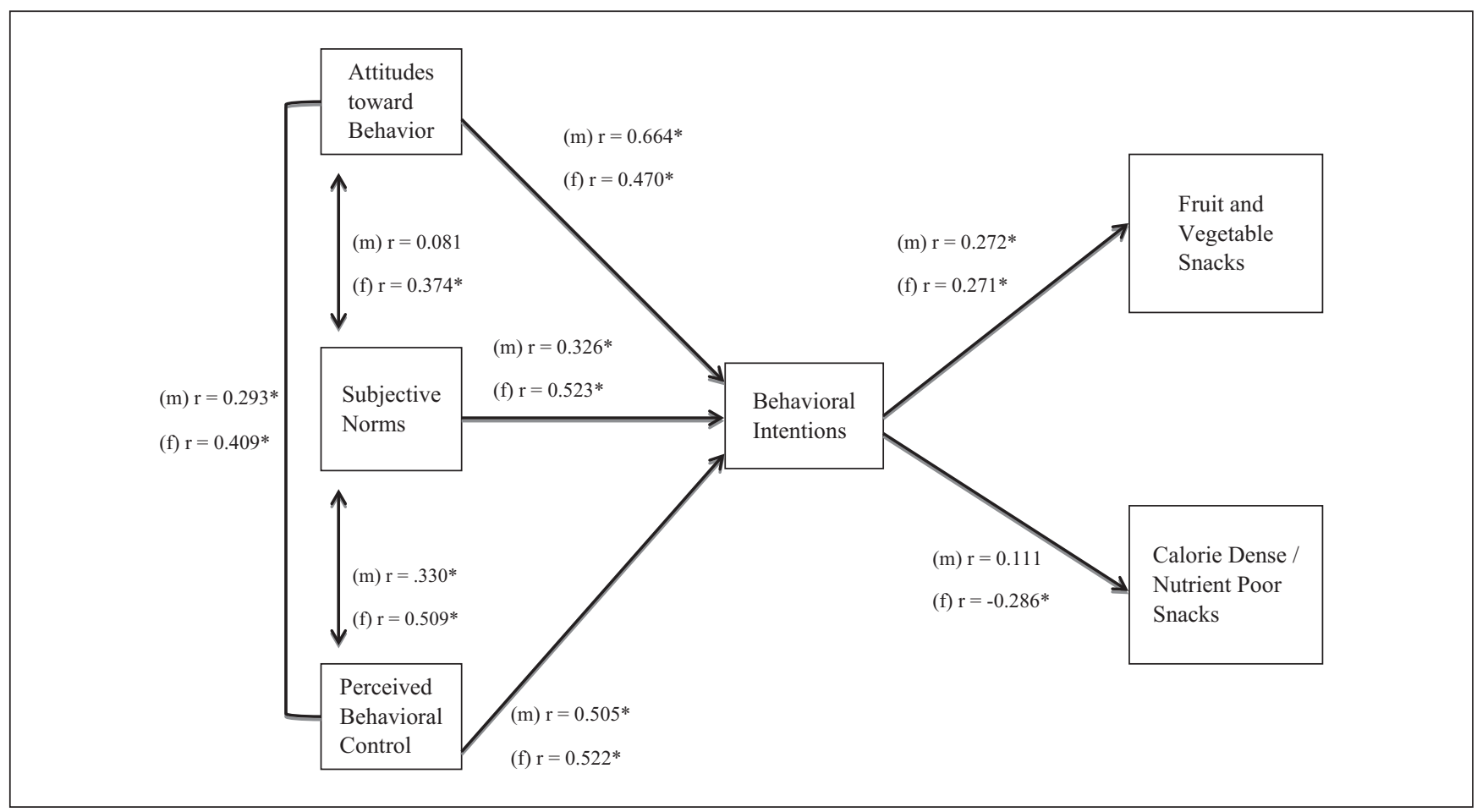

FIGURE 1 Pearson Correlation Coefficients Among Constructs of the Theory of Planned Behavior and Calories From Calorie-Dense/ Nutrient-Poor Snacks and Fruit and Vegetable Snack $(m=$ Male; $f=$ Female) ${ }^{*} p<.01$.

healthy snacks will lead to positive outcomes, such as having a healthy body, leading to a positive anticipated affect. A meta-analysis showed that on average, the inclusion of anticipated affect explains an additional $7 \%$ of the variance of intentions but only an additional $1 \%$ of the variance of behaviors (Sandberg \& Conner, 2008). Although additional variables can be explored and proposed to this theory, Ajzen noted that this task should be taken with caution, and only after careful academic discussion, and sufficient high-quality evidence is produced, should additional predictors be added to the model (Ajzen, 2011).

Behavioral intentions did not predict snacking behaviors well in this study, for either boys or girls. For girls, intentions predicted $6.0 \%$ of the variance for fruit and vegetable snacks $(p=.007)$ and $7.2 \%$ of the variance for calorie-dense/nutrient-poor snacks $(p=.004)$, whereas for boys, intentions predicted $6.4 \%$ of the variance for fruit and vegetable snacks ( $p=.03$ ) but was not significant for calorie-dense/nutrient-poor snacks. These findings are not consistent with a previous metaanalysis, which showed on average intentions predicted $21.2 \%$ of the variance for dietary behaviors. Additionally, the intention-behavior correlation from the meta-analysis was shown to be 0.43 , which was much higher than the correlations found in this study (Figure 1). These findings were similar, however, to other studies predicting dietary behaviors among children and adolescents. In one study using the TPB to predict fruit and vegetable intake and sugar-sweetened beverage intake among adolescents, intention was not significantly related to fruit and vegetable intake and accounted for a very small percentage of the variance $(1.9 \%)$ for sugar-sweetened beverage consumption (Ickes \& Sharma, 2011). Similarly, in another study using Native American youth, in separate analyses for boys and girls, there was no significant relationship between intentions and healthy eating behaviors (Fila \& Smith, 2006).

A number of reasons exist for the discrepancy between intentions and the enactment of both snacking behaviors. First, snack intake may not have been adequately measured, thus resulting in measurement bias. Measuring dietary intake is a difficult task, especially under the circumstances of this study (e.g., the age of the children and setting). Efforts were made to help children be accurate when recalling the previous days snack intake; however, a brief instrument or food 
frequency questionnaire may have been more useful for these circumstances. Additionally, an assumption of this study was that a 1-day recall was representative of normal consumption, which may not have been the case. In the future, researchers should consider using other methods for evaluating dietary behaviors that are representative of "normal" or "typical" snacking behaviors.

Another reason that intentions may not have substantially predicted either snacking behavior was that just as snacking can be infrequent from day to day, intentions can also be unstable. This concept has been coined as intention instability (Fila \& Smith, 2006), where intentions continuously change as the result of external influences, such as environmental cues. Snacking is also likely a behavior that is driven by impulses rather than intentions, which are shaped more so by short-term rewards such as taste, rather than long-term rewards, such as better health.

It has also been noted that behavioral intentions cannot always predict behaviors due to factors that will not allow an individual to act on their intentions. For example, intentions are often moderated by actual control over enacting a behavior (Fishbein, 2008). That is, if children do not truly have control over the types and amounts of snacks that are offered at home or in school, then their intentions cannot be realized and intended behaviors cannot be acted on. It should be noted however, that childhood and adolescence are times of increasing autonomy, as young people transition from eating foods that are given to them with little choice, to having many choices over their diet. Households are also becoming more democratic. One study showed that children as young as 7 and 8 years can have a significant influence on what foods and beverages parents bring into the home environment, thus indirectly controlling what foods are available for snacking (Roberts, Blinkhorn, \& Duxbury, 2003). Results from this study also suggested that a key predictor of young children's dietary autonomy was access to small amounts of money. This was examined in another study, which followed children in the fourth through sixth grade who accessed corner stores before and after school to buy snacks. On average, researchers found that children purchased 2.1 food/beverage items, which averaged to a little more than 350 calories and only cost them $\$ 1.07$ (Borradaile et al., 2009). Therefore, early interventions that can predispose children for making healthier choices, whether they can make them now or in upcoming years, would be likely beneficial. Actual control may also be an important predictor to measure in future studies. It may be argued that this was evaluated as part of the perceived behavioral control, but this is only a proxy measure of actual control, and may not be a reliable measure. Parent surveys are likely best method for evaluating this construct.

Another factor that may have moderated the relationship between intentions and behavior in this study was actual skills and abilities. Skills and abilities were not measured in this study mainly because of logistical concerns. As Buhi, Goodson, Neilands, and Blunt (2011) noted, to measure this construct, skills must be measured objectively, in a naturalistic environment, and is difficult, if not impossible, to measure through self-report. Therefore, more research should focus on this area, and actual control and skills and abilities should be constructs that are operationalized and evaluated in the future by objective means.

There were a number of limitations that should be addressed for this study. Children were obtained from a convenience sample at several Midwestern elementary schools. This limits our ability to generalize results beyond the study participants. This study also was a cross-sectional study by design. Therefore, effects on causality cannot be established. As previously mentioned, all variables in this study were measured by self-report, which inherently can cause misrepresentations in the data based on children being intentionally inaccurate or dishonest. Current knowledge of how snacking impacts childhood obesity is also incomplete. For example, as Nicklas, Baranowski, Cullen, and Berenson (2001) noted, daily meal patterns that consist of snacking (consuming more than three meals per day), have not been consistently associated with obesity among children and adolescents, but this may be because of methodological errors that are common in such studies, such as dietary underreporting. Underreporting has been shown to be a problem especially among energy-dense/nutrient-poor foods (Lafay et al., 2000). According to the American Academy of Nutrition and Dietetics (2012) evidence library, snacking frequency and snack food intake in relation to childhood obesity holds only a Grade III evidence rating, which indicates current evidence is limited to few studies with weak designs. Although it appears more work is needed to resolve this issue, we believe the main issue of concern is replacing unhealthy foods (such as energy-dense/nutrient-poor foods) with healthier alternatives (such as fruits and vegetables), no matter what context the replacement occurs.

This study adds to the current literature by proposing that there are differences in what shapes boys' and girls' intentions toward consuming healthy and unhealthy snack foods. This is an important area of study, because it should not be assumed that interventions for boys and girls are universal, and will be equally effective. As Fishbein and Ajzen (2010) noted, "The relative contribution of attitudes, perceived 
norms, and perceived behavioral control to the prediction of intentions is expected to vary from one person to another, from one group of individuals to another, and from one behavior to another" (p. 180). This is also particularly important when designing public health interventions, because it suggests that boys and girls will likely respond differently to program messages about healthy snacking. According to our findings for boys, modifying attitudes will produce the largest effects for increasing intentions, and for girls, modifying subjective norms will produce the largest effects on intentions. This may also be particular important in settings that are naturally segregated by gender, such as the Boy and Girl Scouts of America, or schools in which only one gender attends.

\section{REFERENCES}

Ajzen, I. (2011). The theory of planned behaviour: Reactions and reflections. Psychology \& Health, 26, 1113-1127.

American Academy of Nutrition and Dietetics. (2012). Is snacking related to adiposity in children? Retrieved from http://www. adaevidencelibrary.com/conclusion.cfm?conclusion_statement _id $=43$ \&highlight $=$ snacking\&home $=1$

Barlow, S. E., \& The Expert Committee. (2007). Expert committee recommendations regarding the prevention, assessment, and treatment of child and adolescent overweight and obesity: Summary report. Pediatrics, 120 (Suppl. 4), S164-S192.

Beadnell, B., Wilsdon, A., Wells, E. A., Morison, D. M., Gillmore, M. R., \& Hoppe, M. (2007). Intrapersonal and interpersonal factors influencing adolescents' decisions about having sex: A test of sufficiency of the theory of planned behavior. Journal of Applied Social Psychology, 37, 2840-2876.

Bere, E., Brug, J., \& Klepp, K. I. (2007). Why do boys eat less fruit and vegetables than girls? Public Health Nutrition, 11, 321-325.

Borradaile, K. E., Sherman, S., Vander Veur, S. S., McCoy, T., Sandoval, B., Nachmani, J., \& Foster, G. D. (2009). Snacking in children: The role of urban corner stores. Pediatrics, 124, 1293-1298.

Branscum, P., \& Sharma, M. (2011-2012). Using the theory of planned behavior to predict two types of snack food consumption among upper elementary children: Implications for practice. International Quarterly of Community Health Education, 32, 41-55.

Buhi, E. R., Goodson, P., Neilands, T. B., \& Blunt, H. (2011). Adolescent sexual abstinence: A test of an integrative theoretical framework. Health Education \& Behavior, 38, 63-79.

Conner, M., \& Mcmillan, B. (1999). Interaction effects in the theory of planned behaviour: Studying cannabis use. British Journal of Social Psychology, 38, 195-222.

Fila, S. A., \& Smith, C. (2006). Applying the theory of planned behavior to healthy eating behaviors in urban Native American youth. International Journal of Behavioral Nutrition and Physical Activity, 3, 11.

Fishbein, M. (2008). A reasoned action approach to health promotion. Medical Decision Making, 28, 834-844.

Fishbein, M., \& Ajzen, I. (2010). Predicting and changing behavior: The reasoned action approach (1st ed.). New York, NY: Psychological Press.
Glanz, K., Rimer, B. K., \& Viswanath, K. (Eds.). (2008). Health behavior and health education: Theory, Research, and Practice (4th ed.). San Francisco, CA: Jossey-Bass.

Grogan, S. C., Bell, R., \& Conner, M. (1997). Eating sweet snacks: Gender differences in attitudes and behaviour. Appetite, 28, 19-31.

Huang, T. T., Howarth, N. C., Lin, B. H., Roberts, S. B., \& McCrory, M. A. (2004). Energy intake and meal portions: Associations with BMI percentile in U.S. children. Obesity Research, 12, 1875-1885. Ickes, M. J., \& Sharma, M. (2011). Does behavioral intention predict nutrition behaviors related to adolescent obesity? ICAN: Infant. Child, \& Adolescent Nutrition, 3, 38-48.

Kassem, N. O., Lee, J. W., Modeste, N. N., \& Johnston, P. K. (2003). Understanding soft drink consumption among female adolescents using the theory of planned behavior. Health Education Research, 18, 278-291.

Lafay, L., Mennen, L., Basdevant, A., Charles, M. A., Borys, J. M., Eschwege, E., \& Romon, M. (2000). Does energy intake underreporting involve all kinds of food or only specific food items? Results from the Fleurbaix Laventie Ville Sante (FLVS) study. International Journal of Obesity and Related Metabolic Disorders, 24, 1500-1506.

Martin, J. J., Oliver, K., \& McCaughtry, N. (2007). The theory of planned behavior: Predicting physical activity in Mexican American children. Journal of Sport \& Exercise Psychology, 29, 225-238.

McEachan, R., Conner, M., Taylor, N. J., \& Lawton, R. J. (2011). Prospective prediction of health-related behaviours with the theory of planned behaviour: A meta-analysis. Health Psychology Review, 5, 97-144.

Nicklas, T. A., Baranowski, T., Cullen, K. W., \& Berenson, G. (2001). Eating patterns, dietary quality and obesity. Journal of the American College of Nutrition, 20, 599-608.

Nosek, B. A., Graham, J., Lindner, N. M., Kesebir, S., Hawkins, C. B., Hahn, C., \& Tenney, E. R. (2010). Cumulative and career-stage citation impact of social-personality psychology programs and their members. Personality and Social Psychology Bulletin, 36, 1283-1300.

Ogden, C. L., Carroll, M. D., Curtin, L. R., Lamb, M. M., \& Flegal, K. M. (2010). Prevalence of high body mass index in US children and adolescents, 2007-2008. Journal of the American Medical Association, 303, 242-249.

Ogden, C. L., Carroll, M. D., \& Flegal, K. M. (2008). High body mass index for age among US children and adolescents, 20032006. Journal of the American Medical Association, 299, 24012405.

Ogden, C. L., Carroll, M. D., Kit, B. K., \& Flegal, K. M. (2012). Prevalence of obesity and trends in body mass index among US children and adolescents, 1999-2010. Journal of the American Medical Association, 307, 483-490.

Rise, J., Sheeran, P., \& Hukkelberg, S. (2010). The role of selfidentity in the theory of planned behavior: A meta-analysis. Journal of Applied Social Psychology, 40, 1085-1105.

Roberts, B. P., Blinkhorn, A. S., \& Duxbury, J. T. (2003). The power of children over adults when obtaining sweet snacks. International Journal of Paediatric Dentistry, 13, 76-84.

Sandberg, T., \& Conner, M. (2008). Anticipated regret as an additional predictor in the theory of planned behaviour: A metaanalysis. British Journal of Social Psychology, 47, 589-606. 